\title{
Determination of 6-Monoacetyl-Morphine (6-MAM) in Brain Samples from Heroin Fatalities
}

Gabriella Roda" ${ }^{\text {* }}$, Fiorenza Farè ${ }^{1}$, Lucia Dell'Acqua', Sebastiano Arnoldi', Veniero Gambaro ${ }^{1}$, Antonella Argo ${ }^{2}$, Giacomo Luca Visconti', Eleonora Casagni ${ }^{1}$, Paolo Procaccianti ${ }^{2}$, Marta Cippitelli ${ }^{3}$ and Rino Froldi ${ }^{3}$

${ }^{1}$ Department of Pharmaceutical Sciences, University of Milan, Via Mangiagalli 25, 20133 Milan, Italy

2Institute of Legal Medicine and Insurance, University Hospital, Via Vespers 129, 90127 Palermo, Italy

${ }^{3}$ Institute of Legal Medicine and Insurance, University of Macerata, Via Don Minzoni 9, 62100 Macerata, Italy

\begin{abstract}
Objective: Post-mortem brain samples from 15 deceased patients whose death was heroin related, were analyzed to determine 6-monoacetyl-morphine (6-MAM) concentrations. The samples belonged to people died between 2008 and 2014. The first eight samples were also analyzed in 2012 to determine only morphine and codeine levels.

Method: A GC/MS method was studied in order to enhance sensitivity, thus helping the determination of 6-MAM whose detection is in most cases difficult because of the complexity of the biological matrix. The analytical method was validated using deuterated internal standards (IS-D3, morphine-D3 and codeine-D3) and it showed adequate specificity, linearity, LOD, LOQ precision and accuracy for the determination of the analyte of interest.

Results: 6-MAM was evidenced only in the more recent samples, thus pointing out its low stability. Its concentration ranged from 15.6 to $28.9 \mathrm{ng} / \mathrm{g}$. Morphine and codeine was also determined and a comparison was carried out between the blood and the brain levels of the three analytes. Moreover a parallel was established between the concentrations of morphine and codeine found in the brain in 2012 and 2015.

Conclusion: 6-MAM determination in the brain is particularly important when discriminating between morphine assumption and heroin abuse. In fact in the cases in which it is not detectable in the blood it can be present in the brain. It was noticed that the concentrations of morphine found in the brain in 2015 are higher respect to the levels of 2012; a possible explanation could be that 6-MAM originally present in the brain has hydrolyzed to morphine, thus increasing its levels.
\end{abstract}

Keywords: Heroine; Morphine; Codeine; 6-Acetyl-morphine; Postmortem brain specimen; GC/MS

\section{Introduction}

The concentration of drugs of abuse, which are susceptible to rapid chemical and metabolic hydrolysis, in blood does not always reflects drug concentration at the site of action [1], especially in the case of death for heroin overdose, because post-mortem redistribution or drug instability can result in misleading variations of plasmatic drug levels [2]. In fact the detection of heroin in the biological fluids of consumers is difficult due to its short half-life (2-6 minutes after intravenous injection) [3]. In the body, it is rapidly hydrolyzed to 6-monoacetylmorphine (6-MAM), which in turn is converted into morphine. Subsequently, morphine is conjugated with glucuronic acid to give mainly morphine-3- $\beta$-D-glucuronide and morphine-6- $\beta$-D-glucuronide $[3,4]$. Direct measurements of heroin metabolites concentrations in the brain are useful in post-mortem forensic toxicology to substantiate fatal overdoses [5] because drugs of abuse exert their effects via the central nervous system, so that it can be assumed that the encephalic concentration of these drugs, measured in post-mortem specimens, is close or equal to their perimortem concentration at their site of action [6]. Moreover brain samples show several advantages respect to other specimens because brain is an isolate compartment, endowed with lower metabolic activity, resulting in slower decomposition and delayed process of putrefaction $[7,8]$. On the other hand the brain represents a complex matrix and analysis can be complicated.

In our laboratory, post-mortem samples are routinely subjected to a systematic toxicological analysis (STA) in order to highlight the presence of unknown exogenous compounds (e.g., drugs of abuse, alcohol) that may have played a role in the mechanism of death [9].
In cases in which STA suggests the presence of morphine and codeine, brain specimens are also typically analyzed [4]. In a previous study [10], we developed a gas chromatography/mass spectrometry (GC/MS) method to investigate the brain distribution patterns of morphine and codeine, which was then, applied to the determination of their levels in nuclei specimens from 14 fatal cases of suspected heroin overdose. The results of this analysis were subsequently compared to metabolite levels in the blood obtained from the systematic toxicological analysis (STA) [11]. In this work we focused our attention on the detection of 6-MAM, whose presence is extremely important to discriminate between heroin abuse and therapeutic use of morphine [12-15]. Analyses were performed in fifteen brain samples from heroin fatalities belonging to a period of time between 2008 and 2014 The first eight samples were also analyzed in 2012 [11] to determine morphine and codeine levels. The other seven cases were analyzed for the first time. To this end a modified GC/MS method was studied, applied and validated in order to enhance sensitivity, thus helping the

*Corresponding author: Gabriella Roda, Department of Pharmaceutical Sciences University of Milan, Via Mangiagalli 25, 20133 Milan, Italy, Tel: +39-02-50319328 Fax: +39-02-50319359; E-mail: gabriella.roda@unimi.it

Received November 10, 2015; Accepted December 03, 2015; Published December 07, 2015

Citation: Roda G, Farè F, Acqua LD, Arnoldi S, Gambaro V, et al. (2015) Determination of 6-Monoacetyl-Morphine (6-MAM) in Brain Samples from Heroin Fatalities. Pharm Anal Acta 6: 451. doi:10.4172/21532435.1000451

Copyright: ( 2015 Roda G, et al. This is an open-access article distributed under the terms of the Creative Commons Attribution License, which permits unrestricted use, distribution, and reproduction in any medium, provided the original author and source are credited. 
determination of 6-MAM using deuterated internal standards (IS-D3, morphine-D3 and codeine-D3).

\section{Experimental}

\section{Cases}

Post-mortem samples, which were supplied by the Institute of Legal Medicine and Assurance at the University of Palermo, were collected from 15 deceased patients (Table 1 ). The subjects were mainly men aged between 23 and 56 years (mean 39 years), and circumstantial evidence suggested that the deaths in each case were heroin related. Eight samples (cases 1-8) belonged to people died between 2008 and 2011 and they were analyzed also in 2012 to establish morphine and codeine levels [11]. Seven samples belonged to people died between 2012 and 2014 and they were analyzed for the first time. Nuclei was the brain area withdrawn in all cases as it is representative of the concentration of the analytes in all the brain [9]

All autopsies performed at the Institute of Legal Medicine and Assurance at the University of Palermo was reviewed, and the autopsy documentation, including the charts, final reports and circumstances surrounding each death, were recorded.

Tissue samples from each organ were routinely collected from the subjects during the autopsy and stained with hematoxylin and eosin for histological assessment. During the overall examination of each subject, the cause of death could not be conclusively determined and toxicological analysis was subsequently used to determine it.

\section{Chemicals, reagents and standards}

6-MAM, Morphine, Codeine, Morphine-D3, Codeine-D3 (internal standards, IS-D3) was purchased from S.A.L.A.R.S. (Italy). Methanol and acetonitrile were obtained from Sigma-Aldrich (Germany). Sodium tetraborate/hydrochloric acid ( $\mathrm{pH} \mathrm{9)}$ buffer solution and the mixture BSTFA (N,O-bis(trimethylsilyl) trifluoroacetamide) 99\% and TMCS (trimethylchlorosilane) $1 \%$ were purchased from Fluka (Switzerland); dichloromethane and isopropyl alcohol were obtained from Prolabo (Italy). Ammonium sulfate was supplied by Carlo Erba (Italy), as well as glacial acetic acid. Water $\left(18.2 \mathrm{M} \Omega \cdot \mathrm{cm}^{-1}\right)$ was obtained using a Milli-Q purification system (Millipore, France). All reagents were of analytical grade and stored according to the manufacturers' instructions.
Standards solutions of 6-MAM $(0.1 \mu \mathrm{g} / \mathrm{mL})$, morphine $(0.1 \mu \mathrm{g} /$ $\mathrm{mL})$ and codeine $(0.1 \mu \mathrm{g} / \mathrm{mL})$ were prepared in methanol as well as the internal standard solution (IS-D3; $0.5 \mu \mathrm{g} / \mathrm{mL}$ of morphine-D3 and $0.5 \mu \mathrm{g} / \mathrm{mL}$ of codeine-D3). Every day working standard samples (WSS) containing $20 \mathrm{ng}_{\text {tot }}$ of 6-MAM, $100 \mathrm{ng}_{\text {tot }}$ of morphine and $100 \mathrm{ng}_{\text {tot }}$ of codeine were prepared in methanol.

\section{Brain tissue sample preparation and deproteinization}

Each sample was homogenized by blending or ball milling (depending on the quantity of the material available) and deproteinized via an ultrasonic bath using the following protocol: $500 \mathrm{mg}$ brain tissue and $100 \mu \mathrm{l}$ IS-D3 were diluted with $4 \mathrm{ml}$ water and $2 \mathrm{ml} \mathrm{pH}$ 9 buffer solution. The resulting mixture was sonicated for $15 \mathrm{~min}$ at room temperature. After centrifugation ( $4000 \mathrm{rpm}, 5 \mathrm{~min}$ ), the clear supernatant was separated and extracted via SPE.

\section{Extraction procedure}

Homogenized and deproteinized encephalic samples were extracted using Bond Elut-LRC Certify solid phase extraction cartridges (Varian, Palo Alto, CA, US) with a Varian vacuum manifold (Varian, CA, USA). Cartridges were first conditioned with $2 \mathrm{ml}$ methanol and $2 \mathrm{ml} \mathrm{pH} 9$ buffer solutions. The supernatants resulting from the centrifugation were loaded on to the cartridges and permitted to absorb through gravity. The cartridges were then washed with $2 \mathrm{ml}$ water, $3 \mathrm{ml}$ of $1 \mathrm{M}$ hydrochloric acid and $0.5 \mathrm{ml}$ methanol. The analytes were subsequently eluted with $2 \times 1 \mathrm{ml}$ elution solvent (dichloromethane/isopropyl alcohol/ammonium hydroxide, $8 / 2 / 0.2, v / v / v)$.

\section{Chromatography}

The extracts were evaporated to dryness under a stream of nitrogen at $40^{\circ} \mathrm{C}$ and derivatized with a mixture of BSTFA (N,O-bis(trimethylsilyl) trifluoroacetamide) $99 \%$ and TMCS (trimethylchlorosilane) $1 \%$ $(50 \mu \mathrm{l})$ at $70^{\circ} \mathrm{C}$ for $30 \mathrm{~min}$. GC/MS analysis was performed on a GC 6890 Plus with a mass selective detector and 6890 autosampler. Data were analyzed with MSD ChemStation D.03.00 software (Agilent Technologies). Chromatographic separation was carried out on a RXI5silMS (G59) capillary column $(30 \mathrm{~m} \times 0.25 \mathrm{~mm}$ I.D., thickness 0.25 $\mu \mathrm{m}$; Restek Bellefonte PA, US).

The GC/MS system was operated under the following conditions: injection temperature: $280^{\circ} \mathrm{C}$ (splitless mode; 0.25 min splitless

Table 1: Overview of the cases studied and concentration of 6-MAM, morphine (Mor) and codeine (Cod) in the brain (ng/g) and in the blood (ng/mL) from STA [11].

\begin{tabular}{|c|c|c|c|c|c|c|c|c|c|}
\hline \multirow{2}{*}{ Case } & \multirow{2}{*}{ Age } & \multirow{2}{*}{ Gender } & \multirow{2}{*}{ Year of death } & \multicolumn{3}{|c|}{ Brain (ng/g) } & \multicolumn{3}{|c|}{ Blood (ng/mL) } \\
\hline & & & & 6-MAM & Mor & Cod & 6-MAM & Mor & Cod \\
\hline 1 & 23 & $M$ & 2008 & $<$ LOD & 175.2 & 23.5 & $<$ LOD & 33 & 10 \\
\hline 2 & 24 & $M$ & 2009 & $<$ LOD & 165.5 & 35.4 & $<$ LOD & 315 & 73 \\
\hline 3 & 42 & $M$ & 2009 & $<$ LOD & 525.5 & 83.7 & $<$ LOD & 338 & 64 \\
\hline 4 & 45 & M & 2009 & $<L O D$ & 534.5 & 44.3 & $<\mathrm{LOD}$ & 688 & 150 \\
\hline 5 & 36 & $M$ & 2009 & $<$ LOD & 149.4 & 20.6 & $<$ LOD & 615 & 193 \\
\hline 6 & 45 & $M$ & 2009 & $<$ LOD & 241.5 & 47.0 & $<$ LOD & 228 & 25 \\
\hline 7 & 42 & $M$ & 2011 & $<$ LOD & 158.4 & 23.7 & $<$ LOD & 130 & $<L O D$ \\
\hline 8 & 35 & $M$ & 2011 & $<$ LOD & 132.5 & 17.7 & $<$ LOD & 171 & $<L O D$ \\
\hline 9 & 40 & $\mathrm{~F}$ & 2012 & $<$ LOD & 157.4 & $<$ LOD & $<$ LOD & 117 & 9.1 \\
\hline 10 & 56 & $M$ & 2013 & 15.6 & 273.0 & 23.3 & 6.1 & 265 & 19.8 \\
\hline 11 & 38 & $M$ & 2013 & 20.5 & 375.2 & 35.6 & $<$ LOD & 475 & 28.8 \\
\hline 12 & 48 & $M$ & 2013 & 28.9 & 246.4 & 25.4 & $<$ LOD & 441 & 29.1 \\
\hline 13 & 35 & $M$ & 2014 & 24.6 & 157.1 & 20.2 & $<L O D$ & 192 & 14.6 \\
\hline 14 & 43 & $M$ & 2014 & $<$ LOD & 147.7 & 10.0 & $<$ LOD & 78 & 11.3 \\
\hline 15 & 30 & $M$ & 2014 & 20.9 & 928.6 & 138.0 & $<$ LOD & 764 & 85 \\
\hline
\end{tabular}


time); interface transfer line: $280^{\circ} \mathrm{C}$; ion source: $230^{\circ} \mathrm{C}$; initial column temperature: $70^{\circ} \mathrm{C}$. The temperature was subsequently increased to $180^{\circ} \mathrm{C}$ at a rate of $40^{\circ} \mathrm{C} \mathrm{min}^{-1}$, then to $300^{\circ} \mathrm{C}$ at a rate of $10^{\circ} \mathrm{C} \mathrm{min}{ }^{-1}$ and held at this temperature for $5 \mathrm{~min}$. Helium was used as the carrier gas at a flow rate of $1.2 \mathrm{ml} \mathrm{min}^{-1}$. Time of analysis $21 \mathrm{~min}$. MS analysis was performed in SCAN $(50 / 550 \mathrm{~m} / \mathrm{z})$ and SIM mode with a quadrupole mass detector operated in electron ionization mode, with beam energy of $70 \mathrm{eV}$. The injection volume was $1 \mu \mathrm{l}$. The ions selected for SIM mode acquisition were 287, 340, 399 for 6-MAM-TMS; 401, 414, 429 for morphine-2-TMS; 313, 343, 371 for codeine -TMS; 417, 432 for morphine - D3 -2 TMS and 347, 374 for codeine - D3 - TMS (in bold the quantifier ions).

\section{Method validation}

Specificity: Specificity was assessed by extracting control blank brain samples in each validation run. The lack of interfering peaks at the same analyte retention times was considered as an acceptable selectivity.

Linearity: The linearity of the response of GC/MS analysis was checked for 6-MAM, codeine and morphine by plotting drug/internal standard peak area ratios versus the total amount of drug in the standard solutions, in the interval 10-1000 ng. Linearity was assessed for each analyte in a specific range preparing WSS at a concentration of 10, 15, 20, 50, $100 \mathrm{ng}_{\text {tot }}$ for 6-MAM; 50, 100, 250, 500, $1000 \mathrm{ng}_{\text {tot }}$ for morphine; 10, 25, 50, 75, $100 \mathrm{ng}_{\text {tot }}$ for codeine. To each standard solution $500 \mathrm{mg}$ of blank brain were added, the mixtures were centrifuged, then $4 \mathrm{~mL}$ of water, $2 \mathrm{~mL}$ of $\mathrm{pH} 9$ buffers and $100 \mu \mathrm{L}$ of IS-D3 were added. The samples were then sonicated for $15 \mathrm{~min}$ and extracted via SPE. Linearity equations were $y=0.0092 x+0.0551\left(R^{2}=0.9905\right)$ for 6-MAM; $\mathrm{y}=0.0072 \mathrm{x}+0.2869 \quad\left(\mathrm{R}^{2}=0.9977\right)$ for morphine; $\mathrm{y}=0.0118 \mathrm{x}-0.0057$ $\left(\mathrm{R}^{2}=0.9815\right)$ for codeine. Calibration curves showed good correlation coefficients for all the analytes over the whole range.

Limit of quantitation (LOQ) and limit of detection (LOD): LOD and LOQ were evaluated analyzing a series of decreasing concentrations of drug-fortified homogenized brain samples. LOD was determined to be the lowest analyte concentration with an $\mathrm{S} / \mathrm{N}$ ratio of at least 3 and resulted $5 \mathrm{ng}$ for 6-MAM, $10 \mathrm{ng}$ for morphine and $5 \mathrm{ng}$ for codeine. LOQ was defined as the lowest concentration with an $\mathrm{S} / \mathrm{N}$ ratio of at least 10 at which the values of accuracy and precision had a coefficient of variation below $15 \%$. The LOQ was $10 \mathrm{ng}$ for 6-MAM and codeine and $25 \mathrm{ng}$ for morphine; it was calculated by analyzing standard solutions of 6-MAM (10 $\left.\mathrm{ng}_{\text {tot }}\right)$, morphine $\left(25 \mathrm{ng}_{\text {tot }}\right)$ and codeine (10 $\mathrm{ng}_{\text {tot }}$ ) during three days.

Accuracy: Accuracy was expressed as the recovery (\%REC) evaluated by analyzing in triplicate five standard solutions for each analyte. In particular 10, 15, 20, 50, $100 \mathrm{ng}_{\text {tot }}$ for 6-MAM; 50, 100, 250, $500,1000 \mathrm{ng}_{\text {tot }}$ for morphine and 10, 25, 50, 75, $100 \mathrm{ng}_{\text {tot }}$ for codeine.

$\% R E C$ was calculated according to: \%REC $=\left[\mathrm{A}_{\text {analyte }} /\left(\mathrm{A}_{\mathrm{IS}} \cdot \mathrm{RR}_{\text {med }} \cdot \mathrm{C}_{\mathrm{t}}\right)\right]$ $\times 100$. Where $C_{t}$ is the total amount of analyte in the standard solution and $\mathrm{RR}_{\text {medium }}$ is defined as follows.

The quantification of the three drugs was based on the response factor $R R$, defined as: $R R=\left(A_{\text {analyye }} / A_{I S}\right) / C_{t}$. Where $A_{\text {analyte }}$ and $A_{\text {IS }}$ are respectively the peak area of the analyte and the peak area of the IS and $C_{t}$ is the analyte concentration in the working standard solution expressed as total ng of analyte. The response factor was calculated for all the working standard solutions described above and the averaged $\mathrm{RR}_{\text {medium }}$ was considered for the estimation of drugs concentration in brain specimens. Accuracy ranged from 70.5 to $120.5 \%$ (mean 100.0\%) for 6-MAM; from 84.7 to $112.4 \%$ for morphine (mean $100.2 \%$ ); from 70.9 to $123.8 \%$ (mean $100.2 \%$ ) for codeine.

Precision: The same standard solutions were analyzed in triplicate during three different days in order to evaluate the precision of the method. The RR value was calculated for every standard solution as well as the medium response ratios $\left(\mathrm{RR}_{\text {medium }}\right)$. Precision was expressed as $\% \mathrm{CV}$. The method showed adequate precision for all the analytes, \%CV being $20.4 \%$ for 6 -MAM, $12.2 \%$ for morphine and $19.1 \%$ for codeine.

\section{Results and Discussion}

In order to evaluate the distribution of morphine and codeine in the brain, in a previous study [11] a GC/MS method was applied to the determination of morphine and codeine levels in nuclei specimens from fourteen fatal cases of suspected heroin overdose. In this work, we focused our attention on the detection of 6-MAM in eight cases considered in the previous paper, of which a sufficient quantity of brain tissue was still available (cases 1-8). In these samples also the levels of morphine and codeine were evaluated. Moreover we analyzed other seven samples from heroin fatalities belonging to a period of time between 2012 and 2014 (cases 9-15). To this end we studied and validated an analytical method suitable to this purpose, using deuterated internal standards in order to enhance sensitivity (Figure 1).

The results obtained from the analysis of the brain samples and a comparison with the blood levels obtained from STA [11] is reported in Table 1.

With regards to the brain tissue samples, a wide distribution of both the concentration of morphine and codeine was noted, which is in agreement with the literature [15].

Even in the blood a high variability was found with regards to the morphine and codeine levels among the fifteen blood samples. As reported in our previous study [11] no relationship between the levels of the two analytes in each individual sample was found, as it is reasonable, being the concentration of codeine linked to the impurities found in "street heroin" (acetyl-codeine).

As stated before particular attention was devoted to the detection of 6-MAM. From the table it is evident that this analyte was evidenced only the more recent brain samples (Figure 2), probably because it undergoes degradation in the brain tissue. In the blood 6-MAM was detected only in one case. The fact that sometimes 6-MAM is detected in the brain and not in the blood is particularly important. This means that when it is not present in the blood, it is mandatory to carry out the determination in the brain tissue before excluding its presence.

In the cases in which analyses of morphine and codeine were carried out either in 2012 or in 2015, we did a comparison between the concentrations of the two analytes (Figure 3 ). It is possible to note that the trend of the analyte concentrations was comparable, even if the analytical method was different and a period of time of three years has elapsed between the two determinations. In the case of morphine, we noticed that the levels determined in 2015 are in some cases higher than those of 2012. This could be due to the hydrolysis of 6-MAM, which was not detected in these samples. To confirm this hypothesis, it could be interesting to analyze cases 9-15 after a few months to see if 6-MAM is still present or it has hydrolyzed to morphine.

\section{Conclusion}

The determination of 6-MAM in the brain is particularly important because it allows the discrimination between heroin assumption and 
Citation: Roda G, Farè F, Acqua LD, Arnoldi S, Gambaro V, et al. (2015) Determination of 6-Monoacetyl-Morphine (6-MAM) in Brain Samples from Heroin Fatalities. Pharm Anal Acta 6: 451. doi:10.4172/21532435.1000451

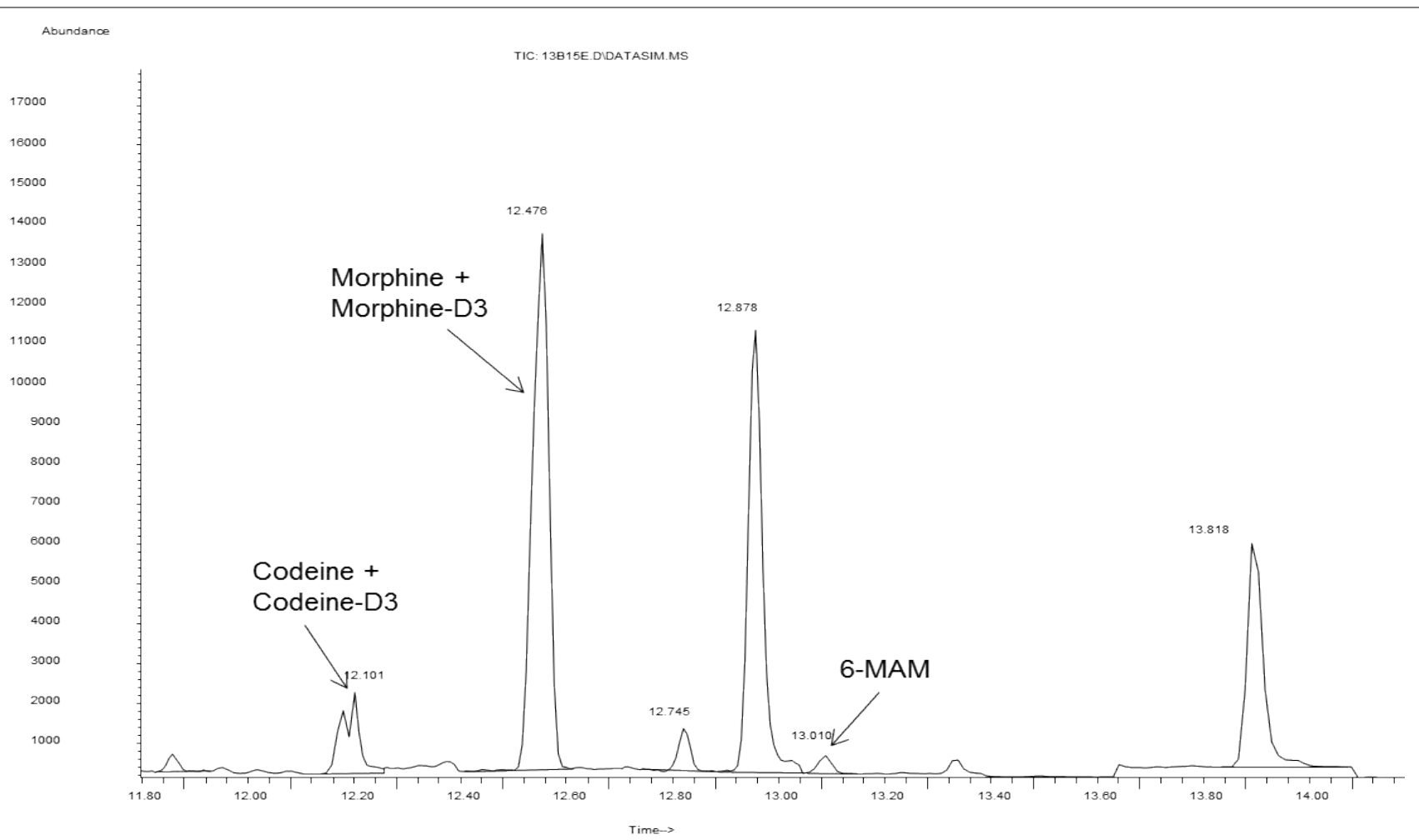

Figure 1: SIM chromatogram of case n. 10.

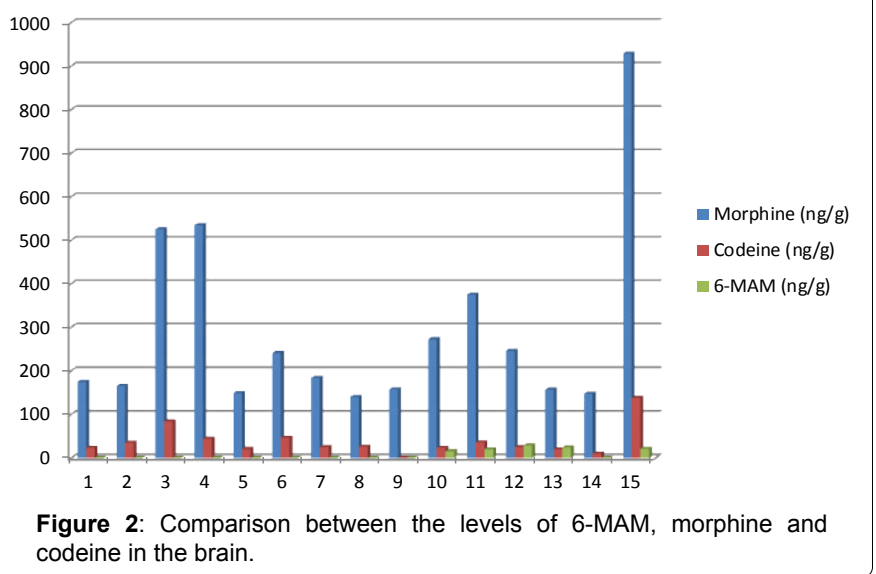

morphine therapeutic use. On the other hand its determination in brain tissue is particularly difficult because of its low concentration and stability. To this end we optimized and validated an analytical method suitable to this purpose, using deuterated IS for quantitative analysis. Together with 6-MAM we carried out the determination of morphine and codeine in the brain and in the blood to compare their levels and try to establish the distribution of heroin metabolites in different biological matrices. A high variability was found for morphine and codeine, while 6-MAM was detected only in the more recent samples confirming its instability in the brain. Eight samples of fifteen were analyzed in 2012 and 2015. The trend of the concentrations of the analytes was confirmed but the morphine levels found in 2015 were higher than those of 2102 . This could be due to the hydrolysis of 6-MAM to morphine. Furthermore in some cases 6-MAM is not

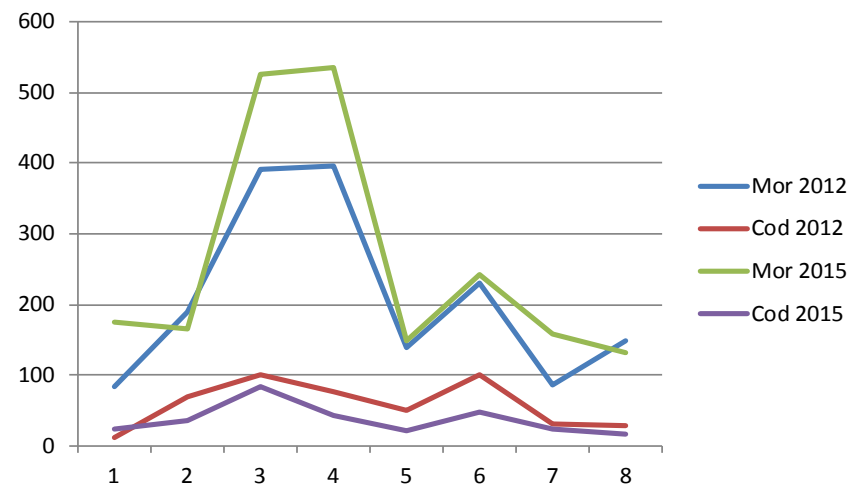

Figure 3: Comparison between the levels (ng/g) of morphine and codeine determined in 2012 and 2015 in the brain.

detectable in the blood but it is present in the brain, thus highlighting the importance of the analysis of this tissue.

\section{References}

1. Lowe R, Barnes A, Lehrmann F, Hyde T, Herman M, et al. (2006) A validated positive chimical ionization GC/MS method for the identification and quantification of amphetamines, opiates, cocaine, and metabolites in human post mortem brain. J Mass Spectr 41: 175-184.

2. Stimpfl T, Reichel S (2007) Distribution of drugs within specific regions of the human brain. Forensic Sci Int 170: 179-182.

3. Baselt RC (2011) Disposition of toxic drugs and chemicals in man (9th edn) Biomedical Publications, Seal Beach, CA.

4. Rook EJ, Huitema ADR, Van den Brink W, Van Ree JM, Beijnen JH (2006) Pharmacokinetics and pharmacokinetic variability of heroin and its metabolites: review of the literature. Curr Clin Pharmacol 1: 109-118. 
Citation: Roda G, Farè F, Acqua LD, Arnoldi S, Gambaro V, et al. (2015) Determination of 6-Monoacetyl-Morphine (6-MAM) in Brain Samples from Heroin Fatalities. Pharm Anal Acta 6: 451. doi:10.4172/21532435.1000451

Page 5 of 5

5. Goldberger BA, Cone EJ, Grant TM, Caplan YH, Levine BS, Smialek JE (1994) Disposition of heroin and its metabolites in heroin related deaths. J Anal Toxicol 18: $22-28$.

6. Jenkins AJ (2008) Drug testing in alternate biological specimens, Humana $\mathrm{Pr}$ Inc 157-180.

7. Moriya F, Hashimoto $Y$ (1996) Post-mortem stability of cocaine and cocaethylene in blood and tissue of human and rabbits. J Forensic Sci 41: 612-616.

8. Sawyer WR, Forney RB (1988) Post-mortem disposition of morphine in rats. Forensic Sci Int 38: 259-273.

9. Sporer KA (1999) Acute heroin overdose. Ann Int Med 130: 584-590.

10. Guerrini K, Argo A, Borroni C, Catalano D, Dell'Acqua L, et al. (2013) Development and validation of a reliable method for studying the distribution pattern for opiates metabolites in brain. J Pharm Biomed Anal 73: 125-130.
11. Gambaro V, Argo A, Cippitelli M, Dell'Acqua L, Farè F, et al. (2014) Unexpected Variation of the Codeine/Morphine Ratio Following Fatal Heroin Overdose. J Anal Toxicol 38: 289-294.

12. Fugelstad A, Ahlner J, Brandt L, Ceder G, Eksborg S, et al. (2003) Use of morphine and 6-monoacetylmorphine in blood for the evaluation of possible risk factors for sudden death in 192 heroin users. Addiction 98: 463-470.

13. Tagliaro F, De Battesti Z (1999) "Heroin overdose" is often the truer description. Addiction 94: 973-974.

14. Kintz $P$, Mangin $P$, Lugnier AA, Chaumont AJ (1989) Identification of 6-monoacetylmorphine, as an indication of heroin abuse. Acta Medicinae Legalis et Socialis (Liege) 39: 464-469.

15. Kintz $P$, Mangin P, Lugnier AA, Chaumont AJ (1989) Toxicological data after heroin overdose. Hum Toxicol 8: 487-489. 